\title{
Scaling properties of multiplicity fluctuations in heavy-ion collisions simulated by AMPT model
}

\author{
Xie Yi-Long, Chen Gang,Wang jiang-ling, Liu Zhao-Hui, Wang Mei-Juan
}

\begin{abstract}
Physics Department, School of Mathematics and Physics, China University of Geosciences Wuhan, China 430074
\end{abstract}

\begin{abstract}
Three dimensional, as well as one- and two-dimensional, studies of multiplicity fluctuation are performed using AMPT model to generate central Au-Au collision events at $\sqrt{s_{\mathrm{NN}}}=200 \mathrm{GeV}$. Two- and three-dimensional normalized factorial moments in rapidity, transverse momentum and azimuthal angle are found to exhibit power-low scaling when partitioning with the same number of bins in each direction, indicating that the fluctuation are isotropic, i.e the fractality is self-similar in multiparticle production of central $\mathrm{Au}-\mathrm{Au}$ collisions. Further, we measured the parameter $\nu$ which it characterizes the intermittency indices derived in particular analysis. It is found that our model result $\nu_{y \varphi p_{t}}=1.86 \pm 0.07$ is larger than $\nu=1.304$, which is the value of Ginzburg-Landau type of phase transition. We also explored the intermittent or fluctuational dependence on the transverse momentum. The result shows that the intermittency or fluctuation increase rapidly with the increasing of transverse momentum.
\end{abstract}

Key words: heavy-ion collisions; multiplicity fluctuations; AMPT model; Scaling properties; self-similar fractal

\section{Introduction}

As has been known, large non-linear fluctuations exist in the process of space-time evolution in high-energy collisions. Such large local fluctuations have been observed in a cosmic-ray event [1] and in high-energy collision experiments [2, 3, 4. Theoretically, the QCD branching cascade [5] involving channels $q \rightarrow q g, g \rightarrow g g$ and $g \rightarrow q \bar{q}$, like other branching processes [6], leads to fractal behavior [7] which manifests itself in the form of power law scaling of final-state multiplicity fluctuations with an increasing resolution in phase space.

In order to be able to decide whether these fluctuations are dynamical, i.e. larger than expected from Poisson noises, Bialas and Peschanski [4] have suggested the use of normalized factorial moments(NFM), which is:

$$
F_{q}(\delta y)=\frac{1}{M} \sum_{m=1}^{M} \frac{<n_{m}\left(n_{m}-1\right) \cdots\left(n_{m}-q+1\right)>}{<n_{m}>^{q}}
$$

where $\delta y=\frac{\Omega}{M}$ is the size of each phase-space when dividing the whole phase space zone $\Omega$ into $M$ parts, and $n_{m}$ is the multiplicity in sub-phase space $\Omega_{m}(\delta y)$. In this way, the dynamic fluctuation in high-energy collisions can be manifested as an abnormal scaling property of NFM, i.e.:

$$
F_{q}(\delta y) \propto(\delta y)^{-\phi_{q}}, \quad(\delta y \rightarrow 0),
$$

when the corresponding collision system is a fractral. In general, for one-dimensional variables, the factorial moments tend to saturate at small phase-space intervals. This can be explained as a projection effect of a three-dimensional phenomenon [8]. It is therefore expected that the scaling phenomenon can be observed in a higher-dimensional analysis.

In three-dimensional phase space the anomalous scaling, or fractal, may be either isotropic or anisotropic, depending on the way a phase space is partitioned [9]. If the scaling is observed when the phase space is partitioned as $\lambda_{a}=\lambda_{b}=\lambda_{c}$, then the anomalous scaling is isotropic (corresponding

\footnotetext{
*Email:chengang1@cug.edu.cn
} 
to a self-similar fractal), where $\lambda_{a}, \lambda_{b}, \lambda_{c}$ denote shrinking ratios of the three phase-space directions; otherwise it is anisotropic (self-affine fractal [6]).

In the 1990s the anomalous scaling of NFM in high-energy collisions were studied extensively [10, 11. In hadron-hadron collisions, the anomalous scaling of NFM was observed 12, 13, to be anisotropic (selfaffine fractal). However, the anomalous scaling of NFM in $\mathrm{e}^{+} \mathrm{e}^{-}$collisions closely obeyed the scaling properties in Eq. (2) for isotropic partition of three-dimensional phase space [14, 15, 16, 17. In our work we applied a self-similar analysis to heavy ion collisions using the Multi-phase Transport (AMPT) model [18] to generate central Au-Au collision events at $\sqrt{s_{\mathrm{NN}}}=200 \mathrm{GeV}$.

In this paper, the AMPT model [18] is introduced in Section 2. The method of self-similar or selfaffine analysis is briefly summarized in Section 3. The results of the self-similar analysis for central $\mathrm{Au}-\mathrm{Au}$ collisions are shown in Section 4. The FM's scaling property and the Ginzburg-Landau type of phase transition applies are discussed in Section 5. Conclusions are presented in Section 6.

\section{A brief introduction of AMPT}

The AMPT model [18 is a mixed model based on both hadronic and partonic phase and exists in two versions: the default AMPT and the AMPT with string melt. Each version contains four subprocesses: phase-space initialization, parton scattering, hadronization and hadron rescattering. The initialization takes the HIJING model as the event generator, which includes minijet production and soft string excitations. Scattering among the partons follows Zhang's Parton Cascade model, which includes only a simple two-body scattering. The cross-sections of the partons are calculated by pQCD. In the default AMPT, the transition from partons to hadrons, i.e. hadronization, follows the Lund string fragmentation model. In this situation, when the partons stop interacting with each other, they melt with their parent strings and are then converted into hadrons. Conversely, in AMPT with string melting, the minijet partons melt with their parent stings to become excited strings, which then fragment into hadrons. Due to the assumption of high initial energy density in the model, these hadrons melt into valence quarks and antiquarks. After the ZPC parton cascade, the hadronization adopts the Quark Coalescence model, in which the two nearest partons combine to become a meson, and three nearest partons into a baryon. Finally, the rescattering and resonance decay of the partons are described by the ART hadronic transport model.

It is well known that the AMPT model with string melting offers a better description of elliptic flow and $\pi$ correlation function, while the default AMPT model provides a better simulation of rapidity distribution and transverse momentum spectrum. In our work, the phase-space variables such as rapidity, transverse momentum and azimuth were used to study the fractal characteristics in central $\mathrm{Au}-\mathrm{Au}$ collisions. We then utilized the default AMPT to generate central $\mathrm{Au}-\mathrm{Au}$ collision events at $\sqrt{s_{\mathrm{NN}}}=200 \mathrm{GeV}$. The impact parameter is in the range $\mathrm{b} \leqslant 2 \mathrm{fm}$ and the parton cross-section is taken to be $3 \mathrm{mb}$.

\section{The method}

In three-dimensional phase space, it can be determined whether the anomalous scaling, or fractal, is isotropic or anisotropic depending on the mode of the phase-space partition. If the scaling, Eq. (2), is observed when the phase space is partitioned according to:

$$
\Delta x_{i} \rightarrow \delta x_{i}=\frac{\Delta x_{i}}{\lambda_{i}},(i=a, b, c)
$$

where $i$ denotes the three phase space directions, then anomalous scaling is isotropic (corresponding to self-similar fractal) in the case of $\lambda_{a}=\lambda_{b}=\lambda_{c}$; otherwise it is anisotropic (self-affine fractal [9]). Note that $\lambda_{i}$ is the phase space representing the partition number or the shrinking ratios in direction $i$. The three-dimensional partition number is the product of the $3 \lambda_{i}$ 's:

$$
M=\lambda_{a} \cdot \lambda_{b} \cdot \lambda_{c} .
$$

The shrinking ratios $\lambda_{a}, \lambda_{b}$ and $\lambda_{c}$ are characterized by a parameter

$$
H_{i j}=\frac{\ln \lambda_{i}}{\ln \lambda_{j}} \quad(i, j=a, b, c),
$$


which is called the Hurst exponent [19]. It is characterized as the isotropic property of a fractal. If all the three Hurst exponents are equal to unity, i.e. $H_{a b}=H_{b c}=H_{c a}=1$, then the fractal is self-similar; otherwise it is self-affine.

The Hurst exponents can be deduced by fitting three one-dimensional, second-order NFM saturation curves [8]:

$$
F_{2}^{i}\left(\lambda_{i}\right)=A_{i}-B_{i} \lambda_{i}^{-\gamma_{i}} \quad(i=a, b, c),
$$

where $\gamma_{i}$ describes the saturation rate of the NFM along the direction of $i$. The Hurst exponents are related to the $\gamma_{i}$ 's as:

$$
H_{i j}=\frac{1+\gamma_{j}}{1+\gamma_{i}} \quad(i, j=a, b, c) .
$$

Therefore, from the final-state multiplicity production of high-energy collisions, we can calculate the Hurst exponents that describe the fractal property of the system, and hence the phase space can be separated properly [20, 21].

However, even in the central region the rapidity distribution is not flat. The particle distribution of final state has a trivial effect on the scaling behavior of the NFM. Therefore, the cumulant variable

$$
X_{c}=\frac{\int_{X_{\min }}^{X} \rho(X) \mathrm{d} X}{\int_{X_{\min }}^{X_{\max }} \rho(X) \mathrm{d} X}
$$

was introduced [22] to reduce the effect of trivial fluctuations, where $X_{c}$ denote $y, p_{t}$ and $\varphi$. In this way, we obtain a flat distribution, i.e. $\rho\left(X_{c}\right)=1$.

\section{The results}

A 5000-event sample for central Au-Au collisions at $\sqrt{s_{\mathrm{NN}}}=200 \mathrm{GeV}$ was produced by the AMPT model. The corresponding results of a self-similar analysis in l-, 2- and 3-dimensional phase space are presented in Figs.1, 2 and 3. The range for the three phase-space variables, rapidity $y$, azimuthal angle $\varphi$ and transverse momentum $p_{t}$, were chosen as $(-6 \leqslant y \leqslant 6,0 \leqslant p t \leqslant 3 \mathrm{GeV}, 0 \leqslant \varphi \leqslant 2 \pi)$.

\subsection{One-dimensional analysis and Hurst parameters}

Fig. 1 shows the results from a one-dimensional analysis of NFM vs. M, which the partitioning $M=1,2, \cdots, 40$ was used for all three variables $\left(M=M_{y}=M_{p_{t}}=M_{\varphi}\right)$. Obviously, the onedimensional second-order NFM saturates for all the three cases of $y, p_{t}$ and $\varphi$.

It is easy to obtain the saturation exponent from Fig. 1, after reducing the influence of momentum conservation [23] by excluding low values of $\mathrm{M}$. The fitting results obtained according to Eq. (6) are also shown in Fig. 1, considering three phase-space variables. The corresponding parameter values are given in Table 1.

Table 1: Parameter values obtained from a fit by Eq. (6) for one-dimensional NFM.

\begin{tabular}{ccccc}
\hline variable & $\mathrm{A}$ & $\mathrm{B}$ & $\gamma$ & $\chi^{2} / D F$ \\
\hline$y$ & $1.001720 \pm 0.000003$ & $0.0008 \pm 0.00003$ & $1.03 \pm 0.04$ & $39 / 37$ \\
$p_{t}$ & $1.001655 \pm 0.000004$ & $0.0004 \pm 0.00003$ & $0.91 \pm 0.07$ & $56 / 37$ \\
$\varphi$ & $1.001521 \pm 0.000004$ & $0.0010 \pm 0.00006$ & $1.03 \pm 0.05$ & $51 / 36$ \\
\hline
\end{tabular}

As shown in Table 1, the saturation index $\gamma_{y}$ and $\gamma_{\varphi}$ are equal, and they are slightly greater than $\gamma_{p_{t}}$. The three exponents are approximately the same, i.e. $\gamma_{y}=\gamma_{\varphi} \approx \gamma_{p_{t}}$, within the error range. Accordingly, the Hurst exponents deduced from Eq. (7) are:

$$
H_{y p_{t}}=0.94 \pm 0.05, \quad H_{\varphi p_{t}}=0.94 \pm 0.06, \quad H_{y \varphi}=1.00 \pm 0.04
$$

From these Hurst exponents, we in fact obtain isotropic values $\left(H_{y p_{t}}=H_{\varphi p_{t}} \approx H_{y \varphi}\right)$ for any two directions of multiparticle production in three-dimensional phase space. This means that fractality in multiparticle production of central $\mathrm{Au}-\mathrm{Au}$ collisions is self-similar. 


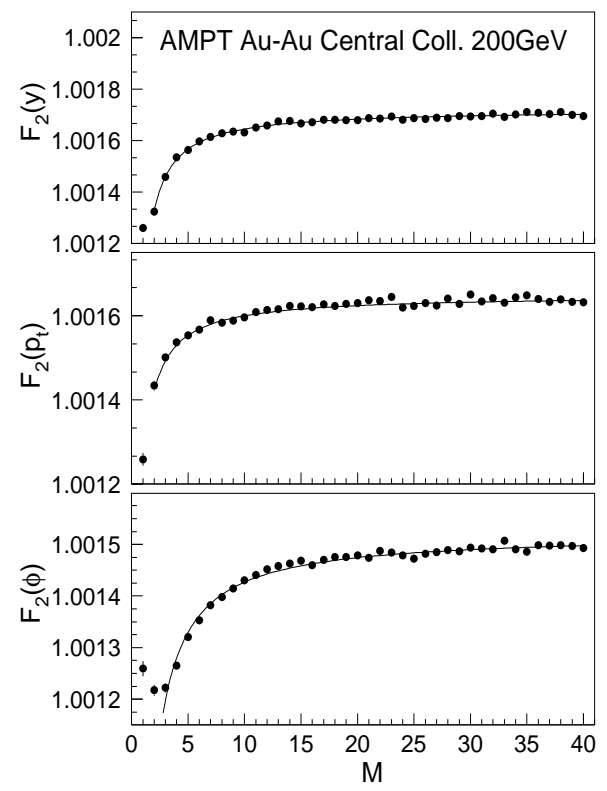

Figure 1: Saturation curves for the second-order NFM of central Au-Au collisions in the three onedimensional variables indicated. The curves are fitted by Eq. (6) after omission of the first point (first two points in the case of $F_{2}(\varphi)$ ).

\subsection{Two-dimensional analysis}

The plots for two-dimensional self-similar NFMs for orders $q=2-9$ in three different planes shown in Fig. 2, which have an isotropic partition of the phase space, i.e. the Hurst exponent by $H_{p_{t} y}=H_{p_{t} \varphi}=H_{y \varphi}=1$. We performed a linear fit to the $\ln F_{q}$ vs. $\ln M$ using:

$$
\ln F_{q}=c+\phi_{q} \ln M,
$$

which is derived from Eq. (2). The $\mathrm{M}=M_{y} M_{\varphi}=M_{\varphi} M_{p_{t}}=M_{y} M_{p_{t}}$ are the partitioning numbers of two-dimensional phase space, and $M_{y}=M_{\varphi}=M_{p_{t}}=1,2, \cdots, 20$ are the partitioning numbers in one dimension. The fitting curves are shown in Fig. 2 as solid lines.

It can be seen that $\ln F_{q}$ increases linearly with increasing $\ln M$, and the plots fit well with a straight line after the first point is omitted in order to reduce the influence of momentum conservation [23]. Similar trends are found in the three planes of the phase space for $(y, \varphi),\left(\varphi, p_{t}\right)$ and $\left(y, p_{t}\right)$ in selfsimilar analysis.

In addition, $F_{q}(y, \varphi)$ presents a better ${ }^{5}$ scaling $^{15}$ feature than ${ }^{20} \stackrel{35}{F}_{q}\left(\stackrel{40}{\varphi}, p_{t}\right)$ or $F_{q}\left(y, p_{t}\right)$, for the reason that the value of Hurst exponents to partition phase space we take to be exactly equal to 1 in all three phase-space planes, but the exponent equals $1\left(H_{y \varphi}=1.00 \pm 0.01\right)$ in the $(y, \varphi)$ plane and is approximately equal to $1\left(H_{y p_{t}}=0.94 \pm 0.05, H_{\varphi p_{t}}=0.94 \pm 0.06\right)$ in the $\left(\varphi, p_{t}\right)$ and $\left(y, p_{t}\right)$ planes. Strictly according to the Hurst exponents in different directions, if we partition the phase space using a non-integer technique [24, 25], the scaling features mpuld, also be all very precise.

\subsection{Three-dimensional analysis}

We can perform a self-similar analysis in three-dimensional phase space, with the Hurst exponents obtained above. For convenience, we approximate the Hurst exponents by $H_{p_{t} y}=H_{p_{t} \varphi}=H_{y \varphi}=1$. From Eq. (3), it follows that $\lambda_{y}=\lambda_{p_{t}}=\lambda_{\varphi}$. We use a partitioning $M=M_{y} M_{p_{t}} M_{\varphi}$, where $M_{y}=$ $M_{p_{t}}=M_{\varphi}=1,2,3, \cdots, 12$.

The results for $\ln F_{q}$ vs. $\ln M$ in three-dimensional phase space for orders $q=2-9$ are shown in Fig. 3. In order to show the quality of the scaling law, linear fits according to Eq. (9) are compared to the data in Fig. 3. The fitting results are listed in Table 2. To reduce the influence of momentum conservation, the first point are excluded in all the fits.

It can be seen from Fig. 3 that the results give a linear fit after the first points for orders $q=2-9$ are omitted. It is pointed out that the fractral of final-state multiplicity production for the central $\mathrm{Au}-\mathrm{Au}$ collision at $\sqrt{s_{\mathrm{NN}}}=200 \mathrm{GeV}$ is self-similar. 


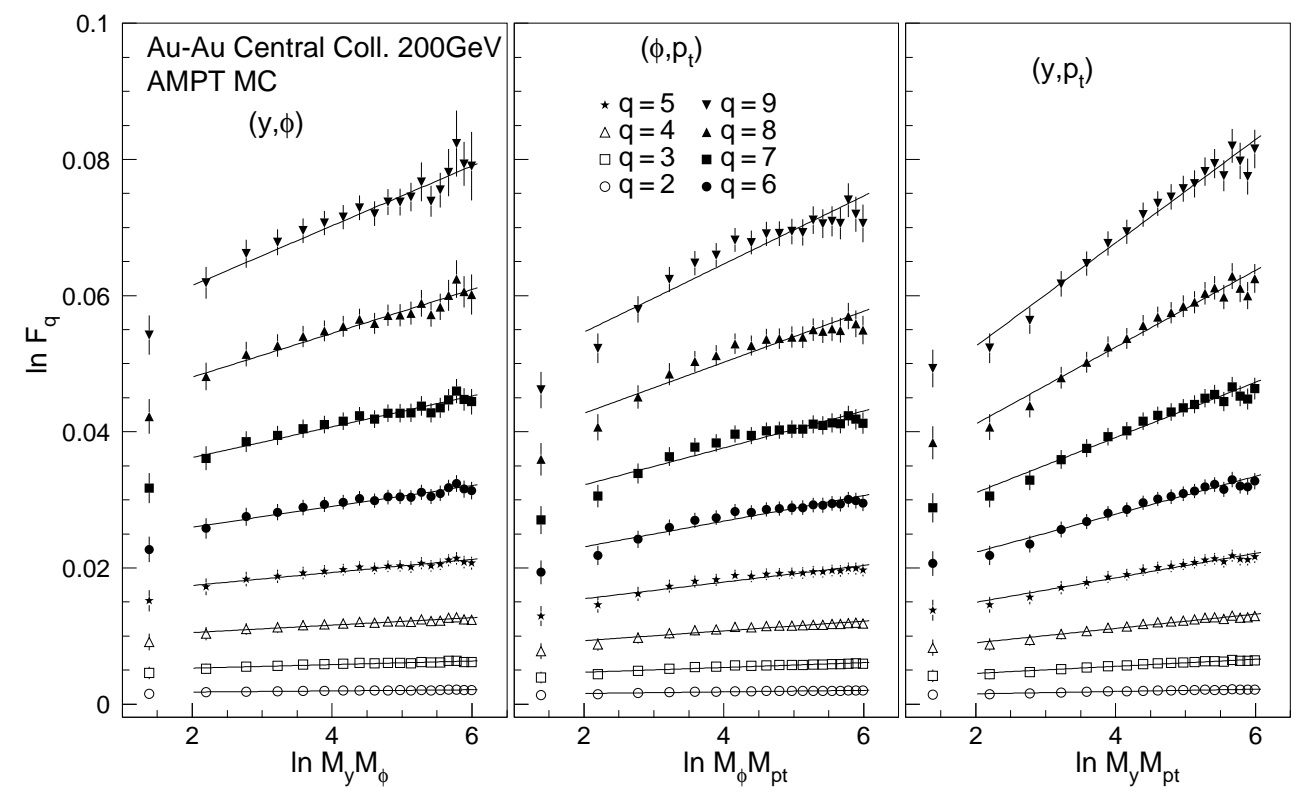

Figure 2: Two-dimensional plots of $\ln F_{q}$ vs. $\ln M$ and the results of the self-similar analysis with a linear fit in central Au-Au collisions. The left-hand figure is for the $(y, \varphi)$ plane, the central figure is for the $\left(\varphi, p_{t}\right)$ plane; the right-hand figure is for the $\left(y, p_{t}\right)$ plane.

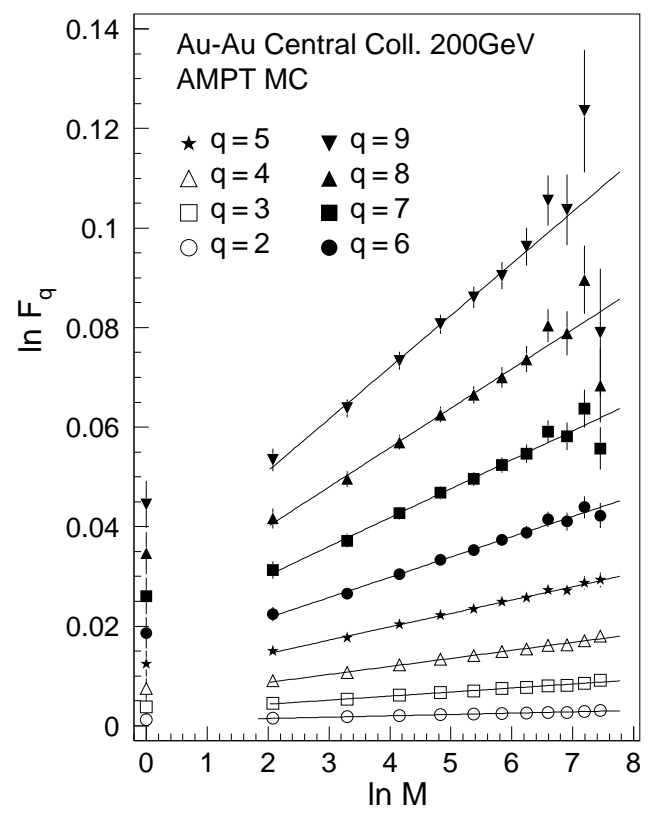

Figure 3: Logarithmic distribution of three-dimensional NFM self-similar analysis in central $\mathrm{Au}-\mathrm{Au}$ collisions at $\sqrt{s_{N N}}=200 \mathrm{GeV}$ for the order $q=2-9$. The curves are fitted by Eq.(9) 
Table 2: Parameter values obtained from a fit of the three-dimensional NFM by Eq. (9).

\begin{tabular}{cccc}
\hline $\mathrm{q}$ & $c$ & $\phi_{q}$ & $\chi^{2} / D F$ \\
\hline 2 & $0.0009 \pm 0.0004$ & $0.0003 \pm 0.0001$ & $0.2 / 8$ \\
3 & $0.0027 \pm 0.0006$ & $0.0008 \pm 0.0001$ & $0.7 / 8$ \\
4 & $0.0055 \pm 0.0009$ & $0.0016 \pm 0.0002$ & $0.8 / 8$ \\
5 & $0.0091 \pm 0.0012$ & $0.0027 \pm 0.0002$ & $0.6 / 8$ \\
6 & $0.0136 \pm 0.0014$ & $0.0041 \pm 0.0003$ & $2 / 8$ \\
7 & $0.0186 \pm 0.0019$ & $0.0058 \pm 0.0004$ & $4 / 8$ \\
8 & $0.0242 \pm 0.0023$ & $0.0079 \pm 0.0005$ & $8 / 8$ \\
9 & $0.0304 \pm 0.0028$ & $0.0104 \pm 0.0006$ & $10 / 8$ \\
\hline
\end{tabular}

However, we can also consider that an effective fluctuation strength can be taken as a characteristic quantity for the strength of dynamical fluctuations [26], defined as:

$$
\alpha_{e f f}=\sqrt{\frac{6 \ln 2}{q}\left(1-D_{q}\right)}=\sqrt{\frac{6 \ln 2}{q} \frac{\phi_{q}}{q-1}},
$$

where $q$ is the order of NFM, and $\phi_{q}$ is the intermittency exponent. We calculate the effective fluctuation strengths $\alpha_{\text {eff }}$ by Eq. (10) in central Au-Au collisions. The results are listed in Table 3. For comparison, Table 3 also lists the effective fluctuation strengths in NA22's hadron-hadron $\left(\pi^{+} p+K^{+} p\right)$ collision experiment at $250 \mathrm{GeV}$ and L3's $\mathrm{e}^{+} \mathrm{e}^{-}$collision experiment at $91.2 \mathrm{GeV}$, where the experimental intermittency exponents for these effective fluctuation strengths are taken from [25, 27, 17].

In Table 3 it can be seen that, firstly, the effective fluctuation strengths are constant when the intermittent exponents increase with the order $q$ increasing; secondly, the effective fluctuation strengths in heavy ion collisions are less than those in hadron-hadron $\left(\pi^{+} p+K^{+} p\right)$ collisions and $\mathrm{e}^{+} \mathrm{e}^{-}$collisions. In other words, the dynamic fluctuation in relativistic heavy ion collisions is much smaller than for hadron-hadron and $\mathrm{e}^{+} \mathrm{e}^{-}$collisions.

Table 3: Comparison of effective fluctuation strengths for hadron-hadron, $\mathrm{e}^{+} \mathrm{e}^{-}$and $\mathrm{Au}-\mathrm{Au}$ collisions.

\begin{tabular}{cccc}
\hline & \multicolumn{3}{c}{$\alpha_{\text {eff }}$} \\
\cline { 2 - 4 } $\mathrm{q}$ & $\mathrm{Au}-\mathrm{Au}$ & $\pi^{+}\left(K^{+}\right) p[25,27]$ & $e^{+} e^{-}[17]$ \\
\hline 2 & $0.025 \pm 0.004$ & $0.356 \pm 0.011$ & $0.635 \pm 0.010$ \\
3 & $0.024 \pm 0.001$ & $0.408 \pm 0.011$ & $0.644 \pm 0.013$ \\
4 & $0.024 \pm 0.001$ & $0.496 \pm 0.012$ & $0.612 \pm 0.009$ \\
5 & $0.024 \pm 0.001$ & $0.572 \pm 0.017$ & $0.600 \pm 0.008$ \\
6 & $0.024 \pm 0.001$ & $*$ & $*$ \\
7 & $0.024 \pm 0.001$ & $*$ & $*$ \\
8 & $0.024 \pm 0.001$ & $*$ & $*$ \\
9 & $0.025 \pm 0.001$ & $*$ & $*$ \\
\hline
\end{tabular}

\section{The discussion of FM's scaling properties}

We can also consider the formula [28]

$$
F_{q} \propto F_{2}^{\beta},
$$

and

$$
\beta_{q} \propto(q-1)^{\nu}
$$

to check scaling property of our $F_{q}$ obtained in the AMPT model, where $\beta_{q}=\phi_{q} / \phi_{2}$. We draw a plot of $\ln F_{q}$ vs $\ln F_{2}$ similar to Fig. 3 shown in the Fig. 4 . In order to show the quality of the scaling law, linear fits according to Eq. (11) are compared to the data in Fig. 4. It is further shown that the system of final-state multiplicity production for the central Au-Au collision at $\sqrt{s_{N N}}=200 \mathrm{GeV}$ exhibits good scaling properties, i.e its fractral is self-similar. The $\nu$ parameter may characterizes all the 


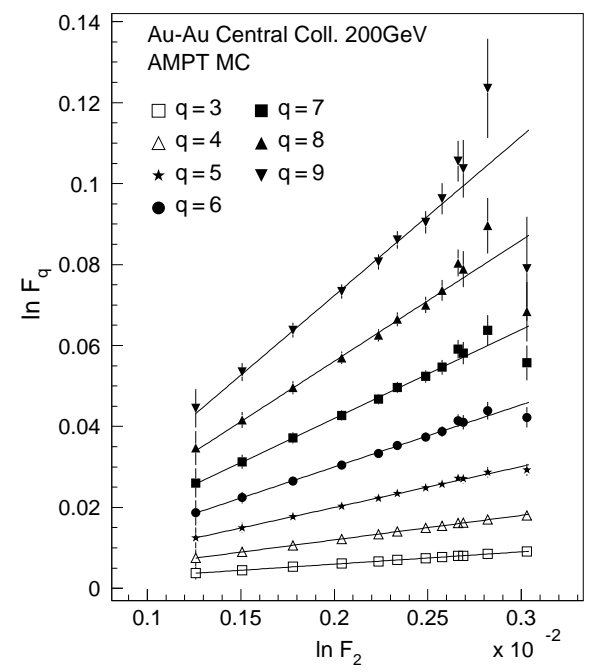

Figure 4: The distribution of 3D $\ln F_{q}$ vs $\ln F_{2}$ in central Au-Au collisions at $\sqrt{s_{N N}}=200 \mathrm{GeV}$. The curves are fitted by Eq.(11)

intermittency indices derived in any particular analysis. If in certain region of phase space where the hadronization of partons created in heavy-ion collisions can be described by the Ginzburg-Landau type of phase transition, then the factorial moments analyzed in that region can be related as in Eq.(12), with $\nu=1.304$ 29]. Using the data from Table 2, a plot of $\beta_{q}\left(\phi_{q} / \phi_{2}\right)$ vs $q$ show in Fig. 5 (solid circle points), with fitting parameter $\nu_{y \varphi p_{t}}=1.86 \pm 0.07$. We also compare the two-dimensional results with the three-dimensional result using same way shown in Fig. 5(the hollow symbols), with the fitting parameters $\nu_{y \varphi}=1.85 \pm 0.13, \nu_{\varphi p_{t}}=1.94 \pm 0.10, \nu_{y p_{t}}=1.94 \pm 0.19$. Obviously, the two-dimensional and three-dimensional results are equal within the error range.

It is noteworthy that there is a big difference between our model result and the value of GinzburgLandau type of phase transition. The fact that $\nu_{y \varphi p_{t}}=1.86 \pm 0.07$ is larger than 1.304 indicates that the fluctuations simulated by AMPT are actually larger than those due to the Ginzburg-Landau type of phase transition, even though Table 3 suggests that those fluctuations are weaker than the ones in hadronic and leptonic collisions. This is a result worthy of our interest in further exploration. But it may be because the AMPT model does not include the physics of phase transition.

It is known that hadronization of partons may occur at different times in the evolution of the system and may populate different $p_{t}$ intervals, depending on the time of hadronization. If we make factorial moment analysis by splitting the $p_{t}$ range to smaller intervals of $0.5 \leqslant p_{t}<1 ., 1 . \leqslant p_{t}<1.5,1.5 \leqslant$ $p_{t}<2.5,2.5 \leqslant p_{t}<3.5,3.5 \leqslant p_{t}<4.5 \mathrm{GeV} / \mathrm{C}$, this different $p_{t}$ slices may avoid the overlapping of multiplicities of hadronization products in an given event, and yield different $\nu$ values. The value of $\nu$ for the $p_{t}$ interval $1 \leqslant p_{t}<1.5 \mathrm{GeV} / \mathrm{C}$ is expected to very different from the value in the interval $3.5 \leqslant p_{t}<4.5 \mathrm{GeV} / \mathrm{C}$ because the latter is dominated by jets effects.

The results of two-dimensional factorial moment loglog distributions in $(y, \varphi)$ plane by splitting the $p_{t}$ range to smaller intervals as given above are shown in Fig.6. One can see that the intermittency or fluctuations obviously increase gradually with the increasing of transverse momentum $p_{t}$ from Fig.6(a) to (b), and then (c), until (e). Nevertheless, the event multiplicity of Au-Au collisions at $\sqrt{s_{N N}}=200 \mathrm{GeV}$ is not high enough to give a well-determined value of $\nu$ for each $p_{t}$ interval. So the quantitative analysis of factorial moment in Fig. 6 can not be made because of the limited statistics of particle numbers per event in the $p_{t}>2.5 \mathrm{GeV} / \mathrm{C}$, as shown in Fig.6(d) and (e).

However, we can consider the factorial moment in the case of phase space partition number $M=1$ as formula

$$
f_{q}(y, \varphi)=\frac{<n_{m}\left(n_{m}-1\right) \cdots\left(n_{m}-q+1\right)>}{<n_{m}>^{q}},
$$

to analyze fluctuational property of final state multiparticle system in $\mathrm{Au}-\mathrm{Au}$ collisions at $\sqrt{s_{N N}}=$ $200 \mathrm{GeV}$. The result of two-dimensional factorial moment in $(y, \varphi)$ plane by splitting the $p_{t}$ range to smaller intervals calculated by Eq.(13) are shown in Fig.7. Obviously, we can see from Fig.7 that the factorial moment $f_{q}(y, \varphi)$ increases rapidly with the increasing of transverse momentum $p_{t}$ indicating 


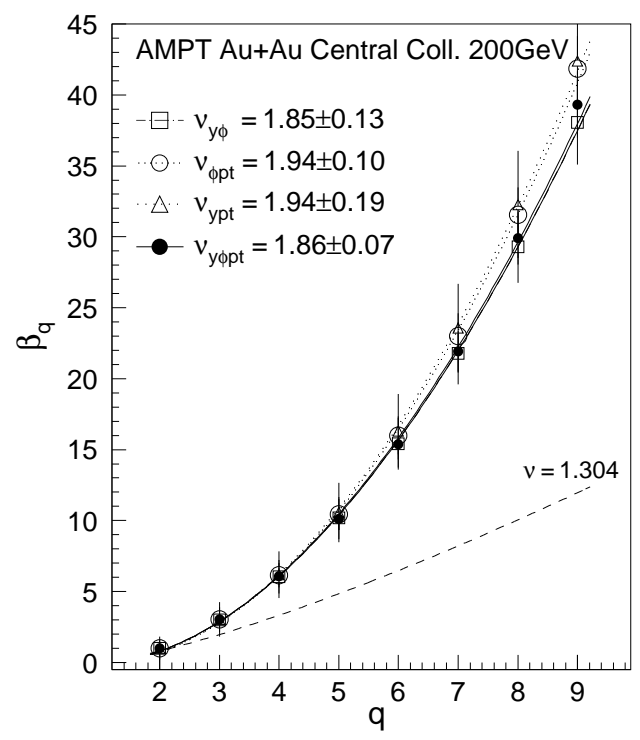

Figure 5: Parameter $\beta_{q}$ vs $q$. The open symbols represent two-dimensional results from Fig.2 and the solid symbols are three-dimensional results from Fig.3. The curves are fitted by Eq.(12). The dashed line corresponds to the case when the Ginzburg-Landau type of phase transition applies.

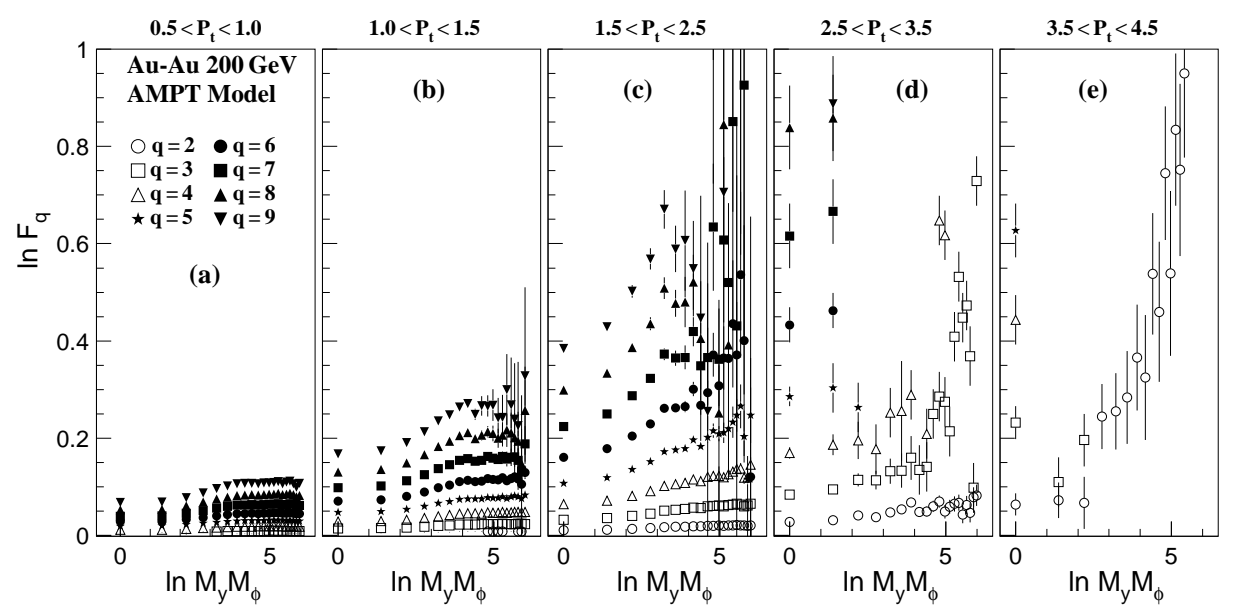

Figure 6: The loglog distribution of two-dimensional factorial moments $F_{q}$ in the $(y, \varphi)$ plane at various $p_{t}$ intervals. 
that the value of fluctuation of final state multiparticle system in $\mathrm{Au}-\mathrm{Au}$ collisions for the $p_{t}$ interval $3.5 \leqslant p_{t}<4.5 \mathrm{GeV} / \mathrm{C}$ is much larger than the value in the interval $1 . \leqslant p_{t}<1.5 \mathrm{GeV} / \mathrm{C}$. These results are consistent with our expectations.

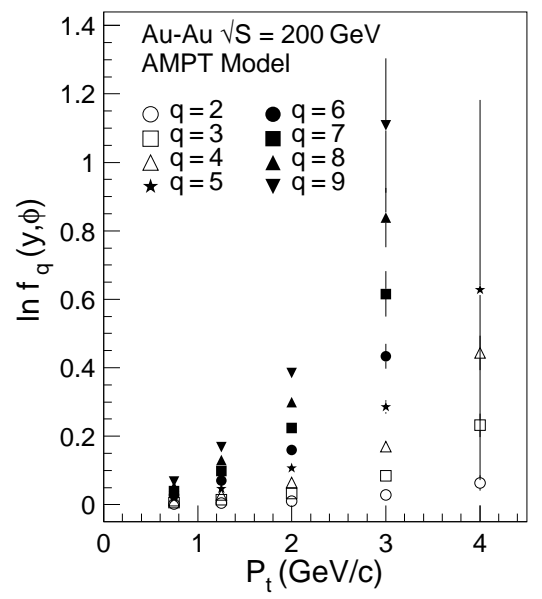

Figure 7: The distribution of two-dimensional factorial moment $\ln f_{q}(y, \varphi)$, as a function of $p_{t}$, with partition number $M=1$.

\section{Summary}

This paper describes a self-similar analysis of the factorial moment for the order $q=2-9$ in threedimensional phase space using the default AMPT model to generate 5000 central Au-Au collisions at $\sqrt{s_{\mathrm{NN}}}=200 \mathrm{GeV}$. By one-dimensional projection of the factorial moments with the Ochs saturation formula Eq. (6), the Hurst exponents were derived for all combinations of the phase-space variables as $\left(y, p_{t}, \varphi\right)$, which are almost identical and approximately equal to 1, i.e. $H_{p_{t} y}=H_{p_{t} \varphi} \simeq H_{y \varphi}=1$. Therefore, we conclude that fractality in multi-particle production of central Au-Au collisions is indeed self-similar. Furthermore, the three-dimensional self-similar analysis shows good scaling behavior.

Finally, the FM's scaling property and the Ginzburg-Landau type of phase transition applies are discussed. We measured the parameter $\nu$ which characterizes the intermittency indices derived in particular analysis. It is found that there is a big difference between our model result $\nu_{y \varphi p_{t}}=1.86 \pm 0.07$ and the value of Ginzburg-Landau type of phase transition $\nu=1.304$. The fact that our model result is larger than 1.304 indicates that the fluctuations simulated by AMPT are actually larger than those due to the Ginzburg-Landau type of phase transition. This is worthy of our interest in further exploration. It may be because the AMPT model does not include the physics of phase transition. We also explored the intermittency and fluctuation in dependence on the transverse momentum. The result shows that the factorial moment, as well as intermittency or fluctuations, increases rapidly with the increasing of transverse momentum $p_{t}$.

It should be noted that our results obtained should be checked by corresponding analysis of the experimental data. If they turn out to disagree, that would indicate the need to modify AMPT, which has been tuned to agree with most features of the data, but not the fluctuations in bin and event multiplicities.

\section{ACKNOWLEDGEMENT}

This work is supported by Fundametal Research Funds for NSFC (11305144, 11303023) and Central Universities (GUGL 100237,120829,130249) in China. The authors thank Professor Wu Yuan-fang and Yang Chun-Bin in the Particle Physics Research Institute of Huazhong Normal University for helpful discussions.

\section{References}

[1] T.H. Burnett et al., JACEE Collabration, Phys. Rev. Lett. 50 (1983) 2062. 
[2] M. Adamus et al., NA22 Collabration, Phys. Lett. B 185 (1987) 200.

[3] P. Carlson, UA5 Collabration, 4th Topical Workshop on pp Collider Physics, March 1983; G.J. Alner et al., UA5 Collabration, Phys. Rep. 154 (1987) 247.

[4] A. Bialas, R. Peschanski, Nucl. Phys. B 273 (1986) 703; A. Bialas, R. Peschanski, Nucl. Phys. B 308 (1988) 857.

[5] Y.L. Dokshitzer et al., Basics of Perturbative QCD, Editions Frontieres, 1991; G. Altarelli, The Development of Perturbative QCD, World Scientific, 1994.

[6] B.B. Mandelbrot, The Fractal Geometry of Nature, W.H. Freeman, 1983; B. B. Mandelbrot, in: F. Family, T. Vicsek (eds.), Dynamics of Fratal Surface, World Scientific, 1991, p. 11.

[7] A. Ukawa, G. Veneziano, Phys. Lett. B 78 (1978) 243; A. Ukawa, G. Veneziano, Nucl. Phys. B 157 (1979) 45.

[8] W. Ochs, Phys. Lett. B 247 (1990)101.

[9] Wu Yuanfang, Liu Lianshou, Phys. Rev. Lett. 70 (1993) 3197.

[10] E.A. De Wolf, I.M. Dremin, W. Kittel, Phys. Rep. 270 (1996) 1.

[11] Wu Yuanfang, Liu Lianshou, Int. J. Mod. Phys. A 18 (2003) 5337.

[12] N. M. Agababyan, et al., NA22 Collabration, Phys. Lett. B 382 (1996) 305.

[13] S.S. Wang ,Z. Wang, C. Wu, Phys. Lett. B 410 (1997) 323.

[14] G. Chen, D.K. Li, L.S. Liu, J. Phys. G 35 (2008) 065002; D.K. Li, G. Chen, H.L. Wei, Chinese Phys. C 65 (2008) 552.

[15] Liu Feng, Liu Fuming, Liu Lianshou, Phys. Rev. D 59 (1999) 114020.

[16] P. Abreu, DELPHI Collabration, Nucl. Phys. B 386 (1992) 471.

[17] Chen Gang, Hu Yuan, Liu Lianshou, et al., in: Bai Yuting, Yu Meiling, Wu Yuanfang (eds.), Proceedings of the Xxxi International Symposium on Multiparticle Dynamics, World Scientific, Singapore, 2002, p. 361.

[18] Zi-Wei Lin, Che Ming Ko, et al., Phys. Rev. C 72 (2005) 064901.

[19] H.E. Hurst, Trans. Amer. Soc. Civil Eng. 116 (1951) 770.

[20] Y.F. Wu, Y. Zhang, L.S. Liu, Phys. Rev. D 51 (1995) 6576.

[21] Y.F. Wu, L.S. Liu, Science in China, A 24 (1994) 1299.

[22] A. Bials, M. Gazdzichi, Phys. Lett. B 252 (1990) 483.

[23] Liu lianshou, Zhang Yang, Deng Yue, Z. Phys. C 73 (1997) 535.

[24] Chen Gang, Liu Lianshou, Gao Yanmin, Int. J. Mod. Phys. A 14 (23) 3687-3697(1999).

[25] N. M. Agababyan, et al., NA22 Collabration, Phys. Lett. B 431 (1998) 451.

[26] Liu Lianshou, Fu Jinghua, Wu Yuanfang, Phys. Lett. B 444 (1998) 563.

[27] I.V. Ajinenko et al., EHS/NA22 Collaboration, Phys. Lett. B 222 (1989) 306.

[28] Rudolph C. Hwa and M. T. Nazirov, Phys. Rev. Lett. 69 (1992) 1741.

[29] Rudolph C. Hwa, Phys. Rev.D 47 (1993) 2773. 